\title{
Convergence of an Automatic hp-Adaptive Finite Element Strategy for Maxwell's Equations
}

\author{
Markus Bürg \\ Preprint Nr. 12/04
}

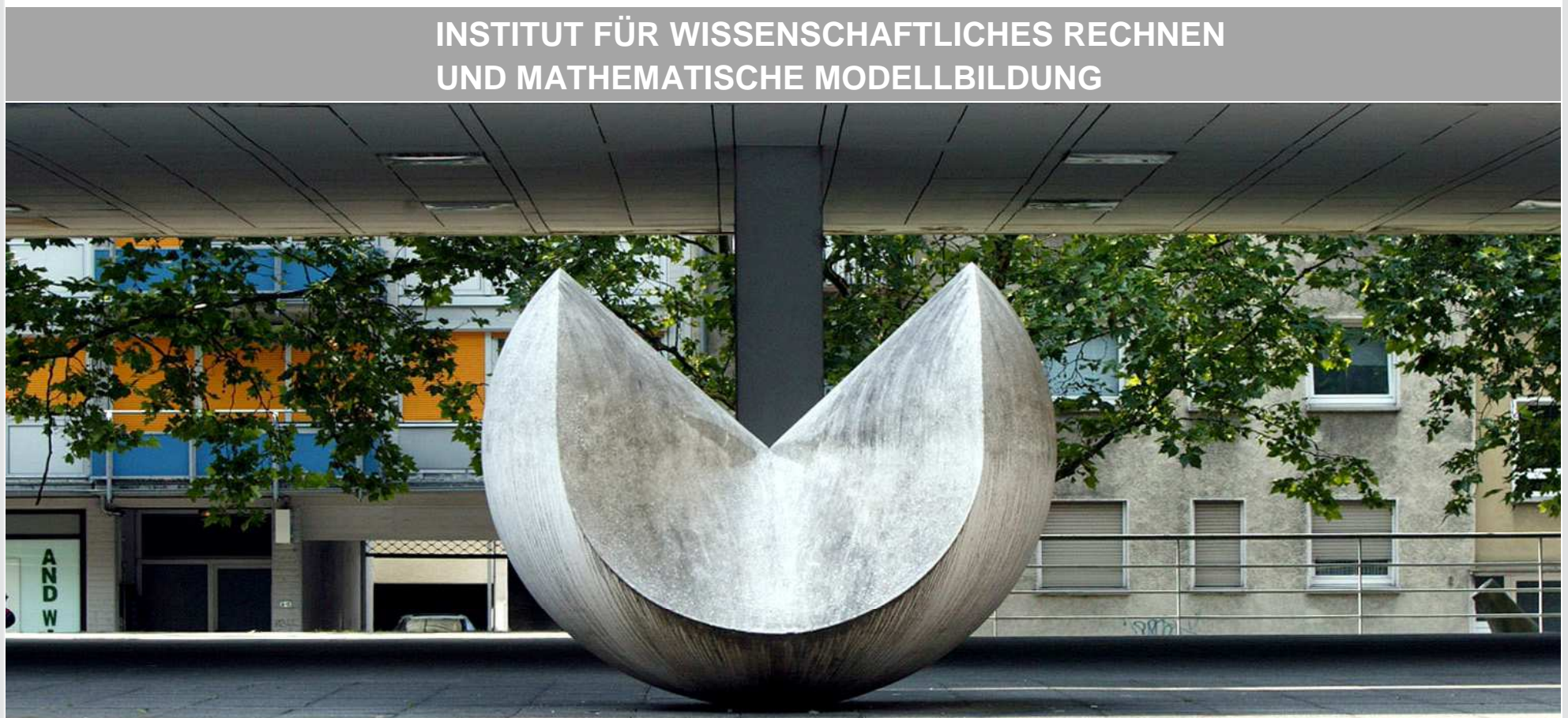

KIT - Universität des Landes Baden-Württemberg und

nationales Forschungszentrum in der Helmholtz-Gemeinschaft 


\section{Anschrift des Verfassers:}

Dipl.-Math. Markus Bürg

Institut für Angewandte und Numerische Mathematik

Karlsruher Institut für Technologie (KIT)

D-76128 Karlsruhe 


\title{
Convergence of an Automatic $h p$-Adaptive Finite Element Strategy for Maxwell's Equations
}

\author{
M. Bürg ${ }^{1}$ \\ Karlsruhe Institute of Technology, Department of Mathematics, D-76128 Karlsruhe
}

\begin{abstract}
We show uniform convergence in the energy norm for an automatic $h p$-adaptive refinement strategy for the finite element method applied to Maxwell's equations.

Keywords: adaptive mesh refinement, a posteriori estimates, convergence, $h p$ version of the finite element method, Maxwell's equations
\end{abstract}

\section{Introduction}

The finite element method is a widely used tool for the numerical solution of partial differential equations. Its performance can be improved by adaptively creating problem-dependent approximation spaces. This can be done by meshrefinement $(h$-FEM) or the use of higher-order ansatz spaces ( $p$-FEM). Taking a combination of both ( $h p$-FEM) can lead to exponentially fast convergence with respect to the number of degrees of freedom [23]. Although in recent years there has been big interest in solving Maxwell's equations numerically, there is only few literature considering the problem-adapted creation of approximation spaces for this system of partial differential equations. The $h$-adaptive finite element method is discussed in e.g. $[3,7,16]$. For the $p$ - and the $h p$-adaptive finite element method Demkowicz, Pardo and co-workers have introduced a global optimization scheme in $[10,12,21,22]$. However this global procedure is computationally quite expensive, since a finer version of the whole problem has to be solved.

In this paper we present an automatic $h p$-adaptive refinement strategy, which is based on the solution of local boundary value problems. Therefore we apply the refinement strategy proposed in $[6,14]$ to Maxwell's equations in the electric field formulation and prove convergence of the adaptive algorithm.

The paper is organized as follows. In Section 2 we introduce the boundary value problem, which we want to consider in this work, and state some general assumptions and notations. The refinement strategy is presented in Section 3

Email address: buerg@kit.edu (M. Bürg)

${ }^{1}$ Tel.: +49-721-608-48091 Fax: +49-721-608-46679 
and in Section 4 we prove its convergence. To conclude the paper we present some numerical examples in Section 5.

\section{Preliminaries}

In this section we introduce Maxwell's equations in the electric field formulation and derive the Dirichlet boundary value problem, which we want to consider here. Then we introduce some basic notations and general assumptions, which we use throughout the paper. To conclude this section the weak formulation of the boundary value problem is derived.

\subsection{Maxwell's equations}

In recent years big interest in the simulation of electromagnetic fields has come up. In many situations these fields can - after exploiting some basic material properties - be described by Maxwell's equations in the electric field formulation $[15,19]$

$$
\begin{aligned}
\frac{d^{2}}{d t^{2}}(\sigma E)+\nabla \times(\alpha \nabla \times E) & =-\frac{d J}{d t} & & \text { in } \Omega \times[0, T] \\
\operatorname{div}(\sigma E) & =0 & & \text { in } \Omega \times[0, T],
\end{aligned}
$$

where $\Omega \subset \mathbb{R}^{3}$ is some connected domain, $E: \Omega \times[0, T] \rightarrow \mathbb{C}^{3}$ denotes the electric field, $\sigma: \Omega \rightarrow \mathbb{C}^{3,3}$ the conductivity, $\alpha: \Omega \rightarrow \mathbb{C}^{3,3}$ the inverse of the magnetic permeability and $J: \Omega \times[0, T] \rightarrow \mathbb{C}^{3}$ the current.

In realistic applications there usually is a sharp distinction between regions, where $\sigma$ can be bounded away from zero, called the conductor, and regions, where $\sigma=0$ holds (cf. Figure 1). Since $\operatorname{div}(J)=0$ for physical reasons, it

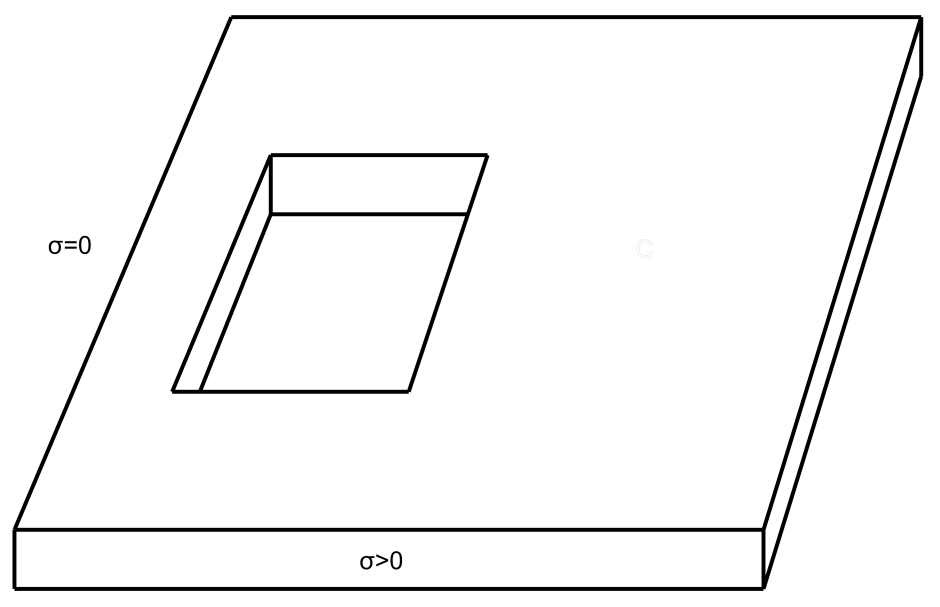

Figure 1: Example for a conductor

follows $\operatorname{div}(\sigma E)=0$ and the first equation of system (1) is sufficient to determine 
the electric field $E$ uniquely inside the conductor. Only outside the conductor the divergence condition is needed. If we drop this condition here, $E$ cannot be determined uniquely anymore. However, the quantity $\nabla \times E$, which usually is the one which we are interested in, is still uniquely determined. Therefore we switch from problem (1) to the formulation

$$
\frac{d^{2}}{d t^{2}}(\sigma E)+\nabla \times(\alpha \nabla \times E)=-\frac{d J}{d t} \quad \text { in } \Omega
$$

and after applying some time stepping-scheme we arrive at the Dirichlet boundary value problem to find $u: \Omega \rightarrow \mathbb{C}^{3}$ such that

$$
\begin{aligned}
\nabla \times(\alpha \nabla \times u)+\beta u & =f & & \text { in } \Omega \\
n \times u & =n \times g & & \text { on } \partial \Omega
\end{aligned}
$$

for given right-hand side function $f: \Omega \rightarrow \mathbb{C}^{3}$ and boundary function $g: \partial \Omega \rightarrow$ $\mathbb{C}^{3}$. Here $f$ depends on $J$ and the values of $u$ from the previous time $\operatorname{step}(\mathrm{s})$ and $\beta$ is given by $\sigma$ scaled with the length of the current time step. From $\operatorname{div}(\nabla \times u)=0$ in $\Omega$ for all sufficiently regular $u: \Omega \rightarrow \mathbb{C}^{3}$ and $\operatorname{div}(f)=0$ in $\Omega$ it follows

$$
\operatorname{div}(\beta u)=0 \quad \text { in } \Omega
$$

\subsection{Notations and assumptions}

Throughout this paper let $\Omega \subset \mathbb{R}^{3}$ be some bounded, open and simplyconnected domain with Lipschitz continuous boundary.

Remark 1. Although, under certain conditions, a generalization of the analysis in this paper into complex space $\mathbb{C}^{3}$ is straightforward, we restrict ourselves to real-valued functions $u$ and coefficients $\alpha$ and $\beta$ for simplicity.

We denote the space of all square integrable functions in $\Omega$ by $L^{2}(\Omega)$ and the space of all essentially bounded functions in $\Omega$ by $L^{\infty}(\Omega)$. Let $\gamma \in \mathbb{N}_{0}^{3}$ be some multi-index and define for $r \geq 0$ the Sobolev spaces $H^{r}(\Omega)$ by

$$
H^{r}(\Omega):=\left\{u \in L^{2}(\Omega): \partial^{\gamma} u \in L^{2}(\Omega) \text { for all } \gamma \text { such that } \sum_{i=1}^{3} \gamma_{i} \leq r\right\}
$$

The space $H($ curl, $\Omega)$ is given by

$$
H(\operatorname{curl}, \Omega):=\left\{u \in L^{2}(\Omega)^{3}: \nabla \times u \in L^{2}(\Omega)^{3}\right\}
$$

and equipped with the norm

$$
\|\cdot\|_{H(\operatorname{curl}, \Omega)}^{2}:=\|\cdot\|_{L^{2}(\Omega)^{3}}^{2}+\|\nabla \times \cdot\|_{L^{2}(\Omega)^{3}}^{2} .
$$

The space of all functions $u \in H(\operatorname{curl}, \Omega)$, which additionally satisfy the homogeneous Dirichlet boundary conditions

$$
n \times u=0 \quad \text { on } \partial \Omega
$$

is dentoted by $H_{0}(\operatorname{curl}, \Omega)$. Further let $\mathcal{K}$ be a triangulation of $\Omega$. Throughout this paper we assume that $\mathcal{K}$ satisfies the following regularity property [23, 25]. 
Definition 1 (Shape regularity). Let $K \in \mathcal{K}$ be the image of reference cell $\widehat{K}$ under some map $F_{K}: \widehat{K} \rightarrow K$ and set $h_{K}:=\operatorname{diam}(K)$. Then $\mathcal{K}$ is $\gamma_{1}$-shape regular, if and only if there exists some constant $\gamma_{1}>0$ such that

$$
\frac{\left\|\nabla F_{K}\right\|_{L^{\infty}(\widehat{K})}}{h_{K}}+h_{K}\left\|\left(\nabla F_{K}\right)^{-1} \circ F_{K}\right\|_{L^{\infty}(\widehat{K})} \leq \gamma_{1} \quad \forall K \in \mathcal{K} .
$$

For $K \in \mathcal{K}$ arbitrary we define the local patch $\omega_{K}$ by

$$
\omega_{K}:=K \cup\{L \in \mathcal{K}: K \text { and } L \text { have a common edge }\} .
$$

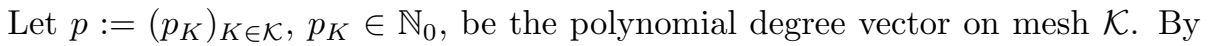
$\widehat{Q}:=[0,1]^{3}$ we denote the reference cube and by

$$
\widehat{T}:=\left\{x \in \mathbb{R}^{3}: 0 \leq x_{1}, x_{2}, x_{3}, x_{1}+x_{2}+x_{3} \leq 1\right\}
$$

the reference tetrahedron. Then the finite-dimensional approximation space of piecewise, vector-valued polynomials is defined by

$$
V_{0}^{p}(\mathcal{K}, \Omega):=\left\{u \in H_{0}(\operatorname{curl}, \Omega):\left(\left.\left(\nabla F_{K}\right)^{-T} u\right|_{K}\right) \circ F_{K} \in P_{p_{K}}(\widehat{K}) \forall K \in \mathcal{K}\right\}
$$

where the polynomial space $P_{p_{K}}(\widehat{K})$ is given by

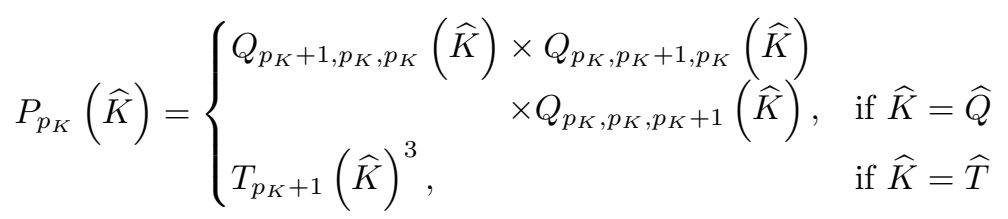

with

$Q_{p, q, r}(\widehat{K})=\operatorname{span}\left\{x_{1}^{i} x_{2}^{j} x_{3}^{k}: x \in \widehat{K}, i \in\{0, \ldots, p\}, j \in\{0, \ldots, q\}, k \in\{0, \ldots, r\}\right\}$

and

$$
T_{p}(\widehat{K})=\operatorname{span}\left\{x_{1}^{i} x_{2}^{j} x_{3}^{k}: x \in \widehat{K}, 0 \leq i+j+k \leq p\right\}
$$

for $p, q, r \in \mathbb{N}_{0}$. Further we define the finite-dimensional approximation space of piecewise, scalar polynomials $X_{0}^{p}(\mathcal{K}, \Omega)$ by

$$
X_{0}^{p}(\mathcal{K}, \Omega):=\left\{\begin{array}{ll}
\left\{q \in H_{0}^{1}(\Omega):\left.q\right|_{K} \circ F_{K} \in Q_{p_{K}+1, p_{K}+1, p_{K}+1}(\widehat{K}) \forall K \in \mathcal{K}\right\}, & \text { if } \widehat{K}=\widehat{Q} \\
\left.q \in H_{0}^{1}(\Omega):\left.q\right|_{K} \circ F_{K} \in T_{p_{K}+1}(\widehat{K}) \forall K \in \mathcal{K}\right\}, & \text { if } \widehat{K}=\widehat{T}
\end{array} .\right.
$$

Then the space of functions $u \in V_{0}^{p}(\mathcal{K}, \Omega)$, which additionally satisfy divergence condition (3) weakly, is denoted by

$$
W_{0}^{p}(\mathcal{K}, \Omega ; \beta):=\left\{u \in V_{0}^{p}(\mathcal{K}, \Omega): \int_{\Omega}(\nabla q)^{T} \beta u=0 \forall q \in X_{0}^{p}(\Omega, \mathcal{K})\right\} .
$$


The local $L^{2}$-projection of the vector-valued function $f$ onto the space of vectorvalued polynomials of degree $p$ is denoted by $f_{p}$. Let $\alpha, \beta: \Omega \rightarrow \mathbb{R}^{3,3}$ be some piecewise matrix-valued polynomials, which are uniformly positive definite, i.e. there exist constants $\alpha_{\max } \geq \alpha_{\min }>0$ and $\beta_{\max } \geq \beta_{\min }>0$ such that for all $u \in L^{2}(\Omega)^{3}$ it holds

$$
\alpha_{\min }\|u\|_{L^{2}(\Omega)^{3}}^{2} \leq \int_{\Omega}\left|u^{T} \alpha u\right| \leq \alpha_{\max }\|u\|_{L^{2}(\Omega)^{3}}^{2}
$$

and

$$
\beta_{\text {min }}\|u\|_{L^{2}(\Omega)^{3}}^{2} \leq \int_{\Omega}\left|u^{T} \beta u\right| \leq \beta_{\max }\|u\|_{L^{2}(\Omega)^{3}}^{2}
$$

a.e. in $\Omega$, respectively.

Remark 2. Note that assumptions (5) and (6) are quite restrictive, but we require these for the analysis of our problem. However, we applied our method successfully to a much broader range of problems, where not all of these assumptions are satisfied.

\subsection{Weak formulation}

To derive the weak formulation of problem (2) we assume that there exists a lifting function $u_{g}: \Omega \rightarrow \mathbb{R}^{3}$ such that

$$
\begin{aligned}
\nabla \times\left(\alpha \nabla \times u_{g}\right)+\beta u_{g} & =0 & & \text { in } \Omega \\
n \times u_{g} & =n \times g & & \text { on } \partial \Omega
\end{aligned}
$$

(cf. [9]). In this case it suffices to find $u: \Omega \rightarrow \mathbb{R}^{3}$ such that

$$
\begin{array}{rlrl}
\nabla \times(\alpha \nabla \times u)+\beta u & =f & & \text { in } \Omega \\
n \times u=0 & & \text { on } \partial \Omega
\end{array}
$$

and adding up the lifting function $u_{g}$ and the solution of problem (7) gives the solution of problem (2). Then we multiply the first equation of (7) by $\phi \in H_{0}(\operatorname{curl}, \Omega)$ and integration by parts yields

$$
\int_{\Omega}(\nabla \times \phi)^{T} \alpha \nabla \times u+\int_{\Omega} \phi^{T} \beta u=\int_{\Omega} \phi^{T} f \quad \forall \phi \in H_{0}(\operatorname{curl}, \Omega) .
$$

Analogously we obtain the discrete problem to find $u_{N} \in V_{0}^{p}(\mathcal{K}, \Omega)$ such that

$$
\int_{\Omega}(\nabla \times \phi)^{T} \alpha \nabla \times u_{N}+\int_{\Omega} \phi^{T} \beta u_{N}=\int_{\Omega} \phi^{T} f \quad \forall \phi \in V_{0}^{p}(\mathcal{K}, \Omega) .
$$

For $u, v \in H_{0}(\operatorname{curl}, \Omega)$ we define the bilinear form $a: H_{0}(\operatorname{curl}, \Omega) \times H_{0}(\operatorname{curl}, \Omega) \rightarrow$ $\mathbb{R}$ by

$$
a(u, v):=\int_{\Omega}(\nabla \times u)^{T} \alpha \nabla \times v+\int_{\Omega} u^{T} \beta v
$$


and the energy norm $\|\cdot\|_{\Omega}: H_{0}(\operatorname{curl}, \Omega) \rightarrow \mathbb{R}_{+}$is given by

$$
\|u\|_{\Omega}^{2}:=a(u, u)
$$

Then one can show that the bilinear form $a$ is elliptic, i.e. for some constant $C_{\text {ell }}>0$ depending on $\alpha_{\min }$ and $\beta_{\min }$ it holds

$$
a(u, u) \geq C_{e l l}\|u\|_{H(\operatorname{curl}, \Omega)}^{2} \quad \forall u \in H_{0}(\operatorname{curl}, \Omega),
$$

and continuous, i.e. for some constant $C_{c}>0$ depending on $\alpha_{\max }$ and $\beta_{\max }$ it holds

$$
|a(u, v)| \leq C_{c}\|u\|_{H(\operatorname{curl}, \Omega)}\|v\|_{H(\operatorname{curl}, \Omega)} \quad \forall u, v \in H_{0}(\operatorname{curl}, \Omega)
$$

(for proofs see for example [19]). Then it follows with the Lax-Milgram Theorem that there exists a unique solution $u \in H_{0}(\operatorname{curl}, \Omega)$ of (8) and a unique solution $u_{N} \in V_{0}^{p}(\mathcal{K}, \Omega)$ of (9) for $f \in L^{2}(\Omega)^{3}$ with $\operatorname{div}(f)=0$ in $\Omega$.

\section{3. $h p$-Adaptive Refinement Strategy}

In this section we present our fully automatic $h p$-adaptive refinement strategy. The refinement strategy is a modification of the adaptive refinement algorithm, which was proposed in $[6,14]$ for simple elliptic equations.

Let TOL $>0$ be some prescribed tolerance. We start the algorithm with a coarse triangulation $\mathcal{K}_{0}$ of $\Omega$ and some finite-dimensional approximation space $V_{0}^{p}\left(\mathcal{K}_{0}, \Omega\right) \subset H_{0}(\operatorname{curl}, \Omega)$. Then we solve discrete problem (9) with this initial configuration and check, if the energy error $\left\|u-u_{N}\right\|_{\Omega}$ is below the prescribed tolerance.

\subsection{Error estimator}

Usually we do not know the analytic solution $u \in H_{0}(\operatorname{curl}, \Omega)$ of (8). Thus we have to estimate the energy error $\left\|u-u_{N}\right\|_{\Omega}$ in terms of the computed solution $u_{N} \in V_{0}^{p}\left(\mathcal{K}_{0}, \Omega\right)$ and the given data. This can be done by computing some reliable a posteriori error estimator $\eta$, which can be bounded in terms of the energy error from above and below with constants independent of mesh size vector $h$ and polynomial degree vector $p$. In [5] the following residualbased a posteriori error estimator for the $h p$-adaptive finite element method for Maxwell's equations was proposed.

Definition 2 (Error estimator). The residual-based a posteriori error estimator $\eta$ is defined as the sum of local error indicators $\eta_{K}, K \in \mathcal{K}$ :

$$
\eta^{2}:=\sum_{K \in \mathcal{K}} \eta_{K}^{2}
$$

For $K \in \mathcal{K}$ the local error indicators $\eta_{K}$ are given by

$$
\eta_{K}^{2}:=\eta_{R, K}^{2}+\eta_{B, K}^{2},
$$


where $\eta_{R, K}$ denotes the residual-based term and $\eta_{B, K}$ denotes the boundary term. The residual-based term $\eta_{R, K}$ is defined by

$$
\eta_{R, K}^{2}:=\frac{h_{K}^{2}}{\left(p_{K}+1\right)^{2}}\left(\left\|\operatorname{res}_{K}\right\|_{L^{2}(K)^{3}}^{2}+\left\|\operatorname{div}\left(\beta u_{N}\right)\right\|_{L^{2}(K)}^{2}\right),
$$

where

$$
\operatorname{res}_{K}:=f_{p_{K}+1}-\nabla \times\left(\alpha \nabla \times u_{N}\right)-\beta u_{N},
$$

and the boundary term $\eta_{B, K}$ by

$$
\eta_{B, K}^{2}:=\sum_{\tilde{f} \subset \partial K \cap \Omega} \frac{h_{\tilde{f}}}{2\left(p_{\tilde{f}}+1\right)}\left(\left\|n_{\tilde{f}} \times\left[\alpha \nabla \times u_{N}\right]\right\|_{L^{2}(\tilde{f})^{3}}^{2}+\left\|n_{\widetilde{f}}^{T}\left[\beta u_{N}\right]\right\|_{L^{2}(\tilde{f})}^{2}\right) .
$$

Here $n_{\tilde{f}}$ denotes the outward-pointing unit normal of cell $K$ on face $\widetilde{f}$ and $[\cdot]$ denotes the jump over the face.

For this a posteriori error estimator the following estimates were shown in $[5]$.

Theorem 1 (A posteriori error estimates). Let $u_{N} \in V_{0}^{p}(\mathcal{K}, \Omega)$ be the solution of discrete problem (9) and $u \in H_{0}(\operatorname{curl}, \Omega) \cap H^{r}(\Omega)^{3}$ be the solution of weak problem (8) for some $\varepsilon>0$ and $r>\frac{1}{2}+\varepsilon$. Further we assume that $\mathcal{K}$ is a $\gamma_{1}$-shape regular triangulation of $\Omega$ and there exists some constant $\gamma_{2}>0$ such that

$$
\frac{p_{K_{1}}+1}{\gamma_{2}} \leq p_{K_{2}}+1 \leq \gamma_{2}\left(p_{K_{1}}+1\right)
$$

for all $K_{1}, K_{2} \in \mathcal{K}$ with $K_{1} \cap K_{2} \neq \emptyset$. Then there exist constants $C_{1}, C_{2}(\varepsilon)>0$ independent of mesh size vector $h$ and polynomial degree vector $p$ such that

$$
\left\|u-u_{N}\right\|_{\Omega}^{2} \leq C_{1} \sum_{K \in \mathcal{K}}\left(p_{K}+1\right)^{2 \varepsilon}\left(\eta_{K}^{2}+\frac{h_{K}^{2}}{\left(p_{K}+1\right)^{2}}\left\|f-f_{p_{K}+1}\right\|_{L^{2}(K)^{3}}^{2}\right)
$$

and

$\eta^{2} \leq C_{2}(\varepsilon) \sum_{K \in \mathcal{K}}\left(p_{K}+1\right)^{2(2+\varepsilon)}\left(\left\|u-u_{N}\right\|_{\omega_{K}}^{2}+\frac{h_{K}^{2}}{\left(p_{K}+1\right)^{2}}\left\|f-f_{p_{K}+1}\right\|_{L^{2}\left(\omega_{K}\right)^{3}}^{2}\right)$.

Proof. See [5], Theorems 5 and 6.

\subsection{Refinement patterns}

A local procedure to enhance the finite element space is called refinement pattern.

For the $h$-adaptive finite element method the common refinement pattern to choose is the equal-weighted bisection in every coordinate direction. If we pick some cell $K \in \mathcal{K}$ and perform an $h$-refinement step, then we probably introduce 
some hanging nodes on the faces and edges of the cell. Thus the boundary contribution of the error would not decay sufficiently. Therefore we also introduce at least some anisotropic refinements on the neighbouring cells, which share at least one edge with cell $K$. This can be seen in Figure 2 on the left-hand side. For the $p$-adaptive finite element method the common choice is to increase the
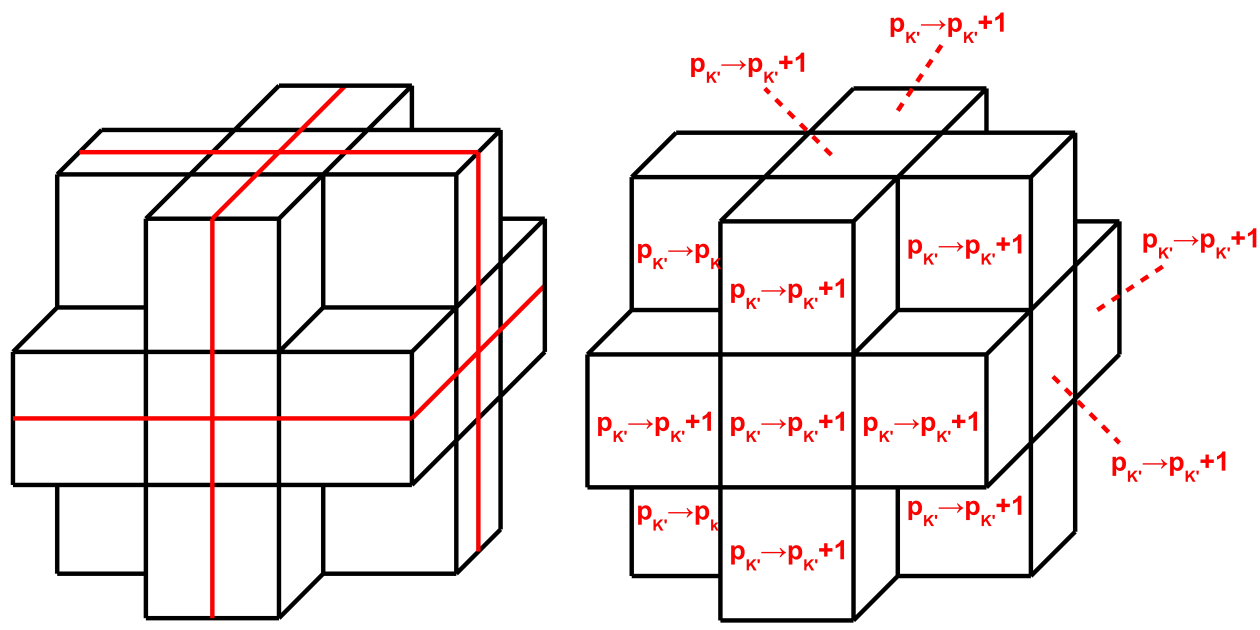

Figure 2: Refinement patterns on cell $K$. Left: Bisection in $x_{1^{-}}, x_{2^{-}}$and $x_{3}$-direction. Right: Increase polynomial degree $p_{K}$ by one.

polynomial degree $p_{K}$ on cell $K$ by one. Also in this case we have to make sure that the boundary contribution of the error is reduced appropriately. Therefore we increase the polynomial degree on all neighbouring cells, which share at least one edge with cell $K$, also by one. This can be seen on the right-hand side of Figure 2.

Thus for the $h p$-adaptive finite element method we have at least two different refinement patterns to choose from, the bisection in every coordinate direction and the increase of the polynomial degree. Of course there are much more refinement patterns, which can be applied here, e.g. anisotropic $h$-refinements, anisotropic $p$-refinements and increase of the polynomial degree by some arbitrary integer $n \in \mathbb{N}$. Thus, without loss of generality we may assume that we have $n \in \mathbb{N}$ with $n \geq 2$ different refinement patterns to choose from.

\subsection{Convergence indicators}

Let $j \in\{1, \ldots, n\}$ and $K \in \mathcal{K}$ be arbitrary. Then we denote the finite element spaces of functions compactly supported in $\omega_{K}$ with refinement pattern $j$ applied to cell $K$ by $V_{0, K, j}^{p}\left(\left.\mathcal{K}\right|_{\omega_{K}}, \omega_{K}\right), W_{0, K, j}^{p}\left(\left.\mathcal{K}\right|_{\omega_{K}}, \omega_{K} ; \beta\right)$ and $X_{0, K, j}^{p}\left(\left.\mathcal{K}\right|_{\omega_{K}}, \omega_{K}\right)$, respectively. Let $\varepsilon_{K, j} \in \mathbb{R}_{+}$be the solution of the optimization problem

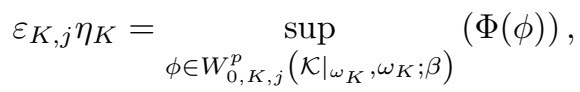


where the mapping $\Phi: W_{0, K, j}^{p}\left(\left.\mathcal{K}\right|_{\omega_{K}}, \omega_{K} ; \beta\right) \rightarrow \mathbb{R}$ is given by

$$
\Phi(\phi):=\frac{\int_{\omega_{K}} \phi^{T} \operatorname{res}_{\omega_{K}}}{\|\phi\|_{\omega_{K}}} .
$$

To solve optimization problem (14) let us consider the following boundary value problem first: Find $v_{j} \in W_{0, K, j}^{p}\left(\left.\mathcal{K}\right|_{\omega_{K}}, \omega_{K} ; \beta\right)$ such that

$$
\int_{\omega_{K}}(\nabla \times \phi)^{T} \alpha \nabla \times v_{j}+\int_{\omega_{K}} \phi^{T} \beta v_{j}=\int_{\omega_{K}} \phi^{T} \operatorname{res}_{\omega_{K}} \quad \forall \phi \in W_{0, K, j}^{p}\left(\left.\mathcal{K}\right|_{\omega_{K}}, \omega_{K} ; \beta\right) .
$$

However for solving this we cannot proceed as we did in Section 2.1 before. Because the residual function res $\omega_{\omega_{K}}$ is unlikely to be weakly divergence-free, this will not hold for the solution $v_{j}$ either, if we do not enforce the condition

$$
\int_{\omega_{K}}(\nabla q)^{T} \beta v_{j}=0 \quad \forall q \in X_{0}^{p}\left(\left.\mathcal{K}\right|_{\omega_{K}}, \omega_{K}\right)
$$

explicitly. Therefore we are now looking for a solution of the following mixed boundary value problem: Find $\left(z_{j}, p_{j}\right) \in V_{0, K, j}^{p}\left(\left.\mathcal{K}\right|_{\omega_{K}}, \omega_{K}\right) \times X_{0, K, j}^{p}\left(\left.\mathcal{K}\right|_{\omega_{K}}, \omega_{K}\right)$ such that

$$
\begin{gathered}
\int_{\omega_{K}}(\nabla \times \phi)^{T} \alpha \nabla \times z_{j}+\int_{\omega_{K}} \phi^{T} \beta z_{j}+\int_{\omega_{K}} \phi^{T} \beta^{T} \nabla p_{j}=\int_{\omega_{K}} \phi^{T} \operatorname{res}_{\omega_{K}} \\
\int_{\omega_{K}}(\nabla \psi)^{T} \beta z_{j}=0
\end{gathered}
$$

for all $\phi \in V_{0, K, j}^{p}\left(\left.\mathcal{K}\right|_{\omega_{K}}, \omega_{K}\right)$ and all $\psi \in X_{0, K, j}^{p}\left(\left.\mathcal{K}\right|_{\omega_{K}}, \omega_{K}\right)$. Then $v_{j}:=z_{j} \in$ $W_{0, K, j}^{p}\left(\left.\mathcal{K}\right|_{\omega_{K}}, \omega_{K} ; \beta\right)$ is the solution of problem (15) and it follows

$$
\begin{aligned}
\Phi(\phi) & =\frac{\int_{\omega_{K}}(\nabla \times \phi)^{T} \alpha \nabla \times v_{j}+\int_{\omega_{K}} \phi^{T} \beta v_{j}}{\|\phi\|_{\omega_{K}}} \\
& \leq \frac{\left\|\alpha^{\frac{1}{2}} \nabla \times \phi\right\|_{L^{2}\left(\omega_{K}\right)^{3}}\left\|\alpha^{\frac{1}{2}} \nabla \times v_{j}\right\|_{L^{2}\left(\omega_{K}\right)^{3}}+\left\|\beta^{\frac{1}{2}} \phi\right\|_{L^{2}\left(\omega_{K}\right)^{3}}\left\|\beta^{\frac{1}{2}} v_{j}\right\|_{L^{2}\left(\omega_{K}\right)^{3}}}{\|\phi\|_{\omega_{K}}}
\end{aligned}
$$

with the Cauchy-Schwarz inequality. We see easily

$$
\begin{aligned}
\Phi(\phi) & \leq\left\|v_{j}\right\|_{\omega_{K}} \\
& =\frac{\int_{\omega_{K}} v_{j}^{T} \operatorname{res}_{\omega_{K}}}{\left\|v_{j}\right\|_{\omega_{K}}} \\
& =\Phi\left(v_{j}\right)
\end{aligned}
$$

and thus $v_{j}$ solves optimization problem (14). 


\subsection{Marking cells for refinement}

As we have seen above, for every cell we get an indication which refinement pattern performs best, if we solve problem (14) for every refinement pattern on every cell. However this information alone might not be enough, because we would like to take into account the work, which is required to achieve the predicted error reduction, too. Therefore we define numbers $w_{K, j}$ to be the number

of degrees of freedom, which the local finite element space $V_{0, K, j}^{p}\left(\left.\mathcal{K}\right|_{\omega_{K}}, \omega_{K}\right)$ has. Then we mark cells for refinement by looking for a solution $\left(\mathcal{A},\left(j_{K}\right)_{K \in \mathcal{A}}\right)$ of the maximization problem

$$
\sum_{K \in \mathcal{A}} \frac{\varepsilon_{K, j_{K}}}{w_{K, j_{K}}}=\max
$$

under the constraint

$$
\sum_{K \in \mathcal{A}}\left(\varepsilon_{K, j_{K}} \eta_{K}\right)^{2} \geq \max _{K \in \mathcal{K}}\left(p_{K}+1\right)^{2 \varepsilon} \theta^{2} \eta^{2},
$$

where $\varepsilon>0$ and $\theta \in(0,1]$ can be chosen arbitrarily. Unfortunately this problem is NP-hard and, hence, it cannot be solved in polynomial time [8]. Therefore we suggest the following strategy to approximate the solution of the maximization problem: In a first step we define the numbers $j_{K} \in\{0, \ldots, n\}$ by

$$
\frac{\varepsilon_{j_{K}}}{w_{j_{K}}}:=\max _{j \in\{1, \ldots, n\}}\left(\frac{\varepsilon_{j}}{w_{j}}\right)
$$

for all $K \in \mathcal{K}$ and then we construct a minimal set $\mathcal{A}$ satisfying constraint (17) by using the SER algorithm, which was presented in [13].

\subsection{The hp-adaptive refinement algorithm}

By combining the steps from above we can now state the fully automatic $h p$-adaptive refinement algorithm for Maxwell's equations. It reads as follows:

(S0) Set up a coarse triangulation $K_{0}$ of $\Omega$ and some polynomial degree vector $p$. Set $N:=0$ and define a tolerance TOL $>0$.

(S1) Solve discrete problem (9) to obtain an approximate solution $u_{N} \in V_{0}^{p}\left(\mathcal{K}_{N}, \Omega\right)$ of problem (8).

(S2) Compute the residual-based error estimator $\eta$. If $\eta<$ TOL: STOP

(S3) Compute the local convergence indicators $\varepsilon_{K, j}$ for all refinement cases $j \in\{1, \ldots, n\}$ and all cells $K \in \mathcal{K}_{N}$.

(S4) Mark the cells for refinement by constructing the tuple $\left(\mathcal{A}_{N},\left(j_{K}\right)_{K \in \mathcal{A}_{N}}\right)$ as proposed in Section 3.4.

(S5) Refine the cells contained in the set $\mathcal{A}_{N}$ according to refinement patterns $\left(j_{K}\right)$. Set $N:=N+1$ and goto step (S1).

Remark 3 (Computing time). Note that in step (S3) the computations for all refinement patterns and all cells are independent of each other. Thus this step can be highly parallelized such that the computing time is reduced significantly in comparison to a sequential approach (cf. Figure 4 on the right-hand side). 


\section{Convergence}

In this section we want to prove convergence of the algorithm presented in Section 3.5. One way to do this is to show that the energy error decays in every refinement step, i.e. for all $N \in \mathbb{N}_{0}$ there exists some constant $\kappa \in(0,1)$ such that

$$
\left\|u-u_{N+1}\right\|_{\Omega} \leq \kappa\left\|u-u_{N}\right\|_{\Omega} .
$$

\subsection{Interpolation}

Before we start with the actual proof let us state an interpolation result, which we need in the proof. This result was shown in [5]. It clarifies the existence of an $H$ (curl)-conforming interpolation operator and gives an upper bound for its interpolation error. For a detailed insight into the construction of the interpolation operator we refer the reader to [5] and [11].

Theorem 2 ( $H$ (curl)-conforming interpolation). Let $K \in \mathcal{K}, \varepsilon>0, r>\frac{1}{2}+\varepsilon$ and $u \in H_{0}(\operatorname{curl}, \Omega) \cap H^{r}(\Omega)^{3}$. Then there exists an interpolation operator $\Pi^{\text {curl }}: H_{0}(\operatorname{curl}, \Omega) \cap H^{r}(\Omega)^{3} \rightarrow V_{0}^{p}(\mathcal{K}, \Omega)$ such that

$$
\left\|\Pi^{\text {curl }} u-u\right\|_{L^{2}(K)^{3}} \leq C_{\text {curl }} \frac{h_{K}^{k}}{\left(p_{K}+1\right)^{r-\varepsilon}}\|u\|_{H^{r}\left(\omega_{K, 1}\right)^{3}}
$$

for some constant $C_{\text {curl }}>0, C_{\text {curl }} \in O\left(\frac{1}{\varepsilon}\right)$ for $\varepsilon \rightarrow 0$, independent of $h_{K}$ and $p_{K}, k:=\min \left\{r, p_{K}+2\right\}$ and

$$
\omega_{K, 1}:=\{L \in \mathcal{K}: K \cap L \neq \emptyset\} .
$$

Proof. See [5], Theorem 3.

\subsection{Discrete Helmholtz decomposition}

With the use of this interpolation operator we can prove the existence and stability of a semi-discrete Helmholtz decomposition for functions from the space $W_{0}^{p}(\mathcal{K}, \Omega ; \beta)$. In [17] a similar result was derived for the lowest-order edge elements.

Lemma 1 (Discrete Helmholtz decomposition). Let $u_{N} \in W_{0}^{p}(\mathcal{K}, \Omega ; \beta)$ and $\varepsilon>0$ arbitrary. Then there exist some $z_{N} \in V_{0}^{p}(\mathcal{K}, \Omega), \zeta_{N} \in X_{0}^{p}(\mathcal{K}, \Omega)^{3}$ and $p_{N} \in X_{0}^{p}(\mathcal{K}, \Omega)$ such that it holds

$$
u_{N}=z_{N}+\Pi^{\mathrm{curl}} \zeta_{N}+\nabla p_{N} .
$$

Further there exists some constant $C_{H}>0$ independent of $h_{K}$ and $p_{K}$ such that

$$
\begin{aligned}
\left\|z_{N}\right\|_{L^{2}(\Omega)^{3}}+\frac{h_{K}}{\left(p_{K}+1\right)^{1-\varepsilon}}\left\|\zeta_{N}\right\|_{H^{1}(\Omega)^{3}}+\left\|\nabla p_{N}\right\|_{L^{2}(\Omega)^{3}} \\
\leq C_{H} \frac{h_{K}}{\left(p_{K}+1\right)^{1-\varepsilon}}\left\|\nabla \times u_{N}\right\|_{L^{2}(\Omega)^{3}} .
\end{aligned}
$$


Proof. In [17], Lemma 5.1, it was shown that there exist some $z_{N} \in V_{0}^{p}(\mathcal{K}, \Omega)$, $\zeta_{N} \in X_{0}^{p}(\mathcal{K}, \Omega)^{3}$ and $p_{N} \in X_{0}^{p}(\mathcal{K}, \Omega)$ such that

$$
u_{N}=z_{N}+\Pi^{\mathrm{curl}} \zeta_{N}+\nabla p_{N} .
$$

Further, Theorem 1.2.3 in [15] implies that there exist some $z \in H_{0}(\operatorname{curl}, \Omega)$ and $p \in H^{1}(\Omega)$ such that

$$
u_{N}=z+\nabla p
$$

with

$$
\operatorname{div}(\beta z)=0 \quad \text { in } \Omega .
$$

Since $u_{N} \in W_{0}^{p}(\mathcal{K}, \Omega ; \beta)$, we have

$$
\begin{aligned}
0 & =\int_{\Omega}(\nabla \psi)^{T} \beta u_{N}=\int_{\Omega}(\nabla \psi)^{T} \beta(z+\nabla p) \\
& =-\int_{\Omega} \psi \operatorname{div}(\beta z)+\int_{\Omega}(\nabla \psi)^{T} \beta \nabla p \\
& =\int_{\Omega}(\nabla \psi)^{T} \beta \nabla p
\end{aligned}
$$

for all $\psi \in X_{0}^{p}(\mathcal{K}, \Omega)$. From [17] we know that there exists some $q \in H^{1}(\Omega)$ such that $p_{N}=p+q$. Then we see

$$
\left\|\nabla p_{N}\right\|_{L^{2}(\Omega)^{3}}^{2} \leq \frac{1}{\beta_{\min }} \int_{\Omega}\left(\nabla p_{N}\right)^{T} \beta \nabla p_{N}
$$

from (6) and it follows

$$
\begin{aligned}
\left\|\nabla p_{N}\right\|_{L^{2}(\Omega)^{3}}^{2} & \leq \frac{1}{\beta_{\min }} \int_{\Omega}\left(\nabla p_{N}\right)^{T} \beta \nabla(p+q) \\
& =\frac{1}{\beta_{\min }} \int_{\Omega}\left(\nabla p_{N}\right)^{T} \beta \nabla q
\end{aligned}
$$

with (18). By the Cauchy-Schwarz inequality we obtain

$$
\begin{aligned}
\left\|\nabla p_{N}\right\|_{L^{2}(\Omega)^{3}}^{2} & \leq \frac{1}{\beta_{\min }}\left\|\beta^{\frac{1}{2}} \nabla p_{N}\right\|_{L^{2}(\Omega)^{3}}\left\|\beta^{\frac{1}{2}} \nabla q\right\|_{L^{2}(\Omega)^{3}} \\
& \leq \frac{\beta_{\max }}{\beta_{\min }}\left\|\nabla p_{N}\right\|_{L^{2}(\Omega)^{3}}\|\nabla q\|_{L^{2}(\Omega)^{3}}
\end{aligned}
$$

with (6). Thus

$$
\left\|\nabla p_{N}\right\|_{L^{2}(\Omega)^{3}} \leq \frac{\beta_{\max }}{\beta_{\min }}\|\nabla q\|_{L^{2}(\Omega)^{3}}
$$

and the estimate follows in exactly the same way as in Lemma 5.1, [17], but replacing the interpolation operator by the one from Theorem 2 . 


\subsection{Convergence theorem}

Now we are ready to prove the convergence of our fully automatic $h p$ adaptive refinement strategy.

Theorem 3 (Convergence). Let $N \in \mathbb{N}_{0}$ be arbitrary and $\mathcal{K}_{N}$ a $\gamma_{1}$-shape regular triangulation of $\Omega$, which satisfies regularity assumption (11). For $r>\frac{1}{2}+\varepsilon$ and $\varepsilon>0$ let $u \in H_{0}(\operatorname{curl}, \Omega) \cap H^{r}(\Omega)^{3}$ be the solution of weak problem (8). Further let $u_{N} \in V_{0}^{p}\left(\mathcal{K}_{N}, \Omega\right)$ and $u_{N+1} \in V_{0}^{p}\left(\mathcal{K}_{N+1}, \Omega\right)$ be the solutions of discrete problem (9) in iteration steps $N$ and $N+1$, respectively. We assume that in every refinement step $N$ the maximization problem (16), (17) has a unique solution $\left(\mathcal{A}_{N},\left(j_{K}\right)_{K \in \mathcal{A}_{N}}\right)$. Let $\theta \in\left(0, \min \left\{1, \sqrt{20 C_{1}}\right\}\right]$ such that

$$
\theta^{2} \geq \frac{5 C_{1} C_{c o v} C_{H}^{2}}{C_{\text {ell }} \max _{K \in \mathcal{K}_{N}}\left(p_{K}+1\right)^{3 \varepsilon}}
$$

with $C_{H}>0$ from Lemma 1, $C_{1}$ from Theorem 1 and $C_{\text {cov }}$ from (24). Additionally we assume that the data error is controlled by the discretization error, i.e. there exists some constant

$$
\mu \in\left(0, \min \left\{1, \frac{1}{\sqrt{2 C_{2}(\varepsilon) C_{\text {cov }}} \max _{K \in \mathcal{K}_{N}}\left(p_{K}+1\right)^{2+\frac{5}{2} \varepsilon}}\right\}\right],
$$

where $C_{2}(\varepsilon)$ is from Theorem 1, such that

$$
\sum_{K \in \mathcal{K}_{N}} \frac{h_{K}^{2}}{\left(p_{K}+1\right)^{2(1-\varepsilon)}}\left\|f-f_{p_{K}+1}\right\|_{L^{2}(K)^{3}}^{2} \leq \mu^{2} \eta^{2} .
$$

Then there exists some constant $\kappa \in(0,1)$ such that

$$
\left\|u-u_{N+1}\right\|_{\Omega} \leq \kappa\left\|u-u_{N}\right\|_{\Omega},
$$

i.e. the energy error decreases in every refinement step of the algorithm from Section 3.5.

Proof. We see

$$
\left\|u-u_{N}\right\|_{\Omega}^{2}=a\left(u-u_{N+1}, u-u_{N}\right)+a\left(u_{N+1}-u_{N}, u-u_{N}\right)
$$

and, since $V_{0}^{p}\left(\mathcal{K}_{N}, \Omega\right) \subset V_{0}^{p}\left(\mathcal{K}_{N+1}, \Omega\right)$, we can use the Galerkin orthogonality to obtain

$$
\left\|u-u_{N}\right\|_{\Omega}^{2}=\left\|u-u_{N+1}\right\|_{\Omega}^{2}+\left\|u_{N+1}-u_{N}\right\|_{\Omega}^{2} .
$$

Thus it suffices to show the existence of some constant $\kappa \in(0,1)$ such that

$$
\left\|u_{N+1}-u_{N}\right\|_{\Omega}^{2} \geq\left(1-\kappa^{2}\right)\left\|u-u_{N}\right\|_{\Omega}^{2} .
$$


Let $K \in \mathcal{A}_{N}$ be arbitrary and $\phi_{N+1} \in W_{0}^{p}\left(\mathcal{K}_{N+1}, \Omega ; \beta\right)$ with $\operatorname{supp}\left(\phi_{N+1}\right) \subset \omega_{K}$.

Since $W_{0}^{p}\left(\mathcal{K}_{N+1}, \Omega ; \beta\right) \subset V_{0}^{p}\left(\mathcal{K}_{N+1}, \Omega\right)$, we have

$$
\begin{aligned}
& \int_{\omega_{K}}\left(\nabla \times \phi_{N+1}\right)^{T} \alpha \nabla \times\left(u_{N+1}-u_{N}\right)+\int_{\omega_{K}} \phi_{N+1}^{T} \beta\left(u_{N+1}-u_{N}\right) \\
& \quad=\int_{\omega_{K}}\left(\left(\nabla \times \phi_{N+1}\right)^{T} \alpha \nabla \times u+\phi_{N+1}^{T} \beta u\right)-\int_{\omega_{K}}\left(\left(\nabla \times \phi_{N+1}\right)^{T} \alpha \nabla \times u_{N}+\phi_{N+1}^{T} \beta u_{N}\right)
\end{aligned}
$$

by Galerkin orthogonality. Then it follows

$$
\begin{aligned}
\int_{\omega_{K}}\left(\nabla \times \phi_{N+1}\right)^{T} \alpha \nabla \times\left(u_{N+1}-u_{N}\right)+\int_{\omega_{K}} \phi_{N+1}^{T} \beta\left(u_{N+1}-u_{N}\right) \\
=\int_{\omega_{K}} \phi_{N+1}^{T}\left(f-\nabla \times\left(\alpha \nabla \times u_{N}\right)-\beta u_{N}\right) \\
=\int_{\omega_{K}} \phi_{N+1}^{T} \operatorname{res}_{\omega_{K}}+\int_{\omega_{K}} \phi_{N+1}^{T}\left(f-f_{{\omega^{K}}_{K}+1}\right)
\end{aligned}
$$

with integration by parts, where

$$
p_{\omega_{K}}:=\max _{L \subset \omega_{K}}\left(p_{L}\right) .
$$

For all $\phi_{N} \in V_{0}^{p}\left(\mathcal{K}_{N}, \Omega\right)$ with $\operatorname{supp}\left(\phi_{N}\right) \subset \omega_{K}$ it holds

$$
\begin{gathered}
\int_{\omega_{K}}\left(\nabla \times \phi_{N+1}\right)^{T} \alpha \nabla \times\left(u_{N+1}-u_{N}\right)+\int_{\omega_{K}} \phi_{N+1}^{T} \beta\left(u_{N+1}-u_{N}\right) \\
=\int_{\omega_{K}} \phi_{N+1}^{T} \operatorname{res}_{\omega_{K}}+\int_{\omega_{K}}\left(\phi_{N+1}-\phi_{N}\right)^{T}\left(f-f_{p_{\omega_{K}}+1}\right)
\end{gathered}
$$

with the definition of $f_{p_{\omega_{K}}+1}$. This implies

$$
\begin{aligned}
& \left|\int_{\omega_{K}}\left(\nabla \times \phi_{N+1}\right)^{T} \alpha \nabla \times\left(u_{N+1}-u_{N}\right)\right|+\left|\int_{\omega_{K}} \phi_{N+1}^{T} \beta\left(u_{N+1}-u_{N}\right)\right| \\
& \quad \geq\left|\int_{\omega_{K}} \phi_{N+1}^{T} \operatorname{res}_{\omega_{K}}\right|-\left|\int_{\omega_{K}}\left(\phi_{N+1}-\phi_{N}\right)^{T}\left(f-f_{p_{\omega_{K}}+1}\right)\right|
\end{aligned}
$$

and by using the Cauchy-Schwarz inequality it follows

$$
\begin{aligned}
\left|\int_{\omega_{K}} \phi_{N+1}^{T} \operatorname{res}_{\omega_{K}}\right| \leq \| & \alpha^{\frac{1}{2}} \nabla \times \phi_{N+1}\left\|_{L^{2}\left(\omega_{K}\right)^{3}}\right\| \alpha^{\frac{1}{2}} \nabla \times\left(u_{N+1}-u_{N}\right) \|_{L^{2}\left(\omega_{K}\right)^{3}} \\
& +\left\|\beta^{\frac{1}{2}} \phi_{N+1}\right\|_{L^{2}\left(\omega_{K}\right)^{3}}\left\|\beta^{\frac{1}{2}}\left(u_{N+1}-u_{N}\right)\right\|_{L^{2}\left(\omega_{K}\right)^{3}} \\
& +\left\|\phi_{N+1}-\phi_{N}\right\|_{L^{2}\left(\omega_{K}\right)^{3}}\left\|f-f_{p_{\omega_{K}}+1}\right\|_{L^{2}\left(\omega_{K}\right)^{3}} .
\end{aligned}
$$

By using the definition of the enery norm we get

$$
\begin{aligned}
\left|\int_{\omega_{K}} \phi_{N+1}^{T} \operatorname{res}_{\omega_{K}}\right| \leq\left\|\phi_{N+1}\right\|_{\omega_{K}}\left\|u_{N+1}-u_{N}\right\|_{\omega_{K}} & \\
& +\left\|\phi_{N+1}-\phi_{N}\right\|_{L^{2}\left(\omega_{K}\right)^{3}}\left\|f-f_{p_{\omega_{K}}+1}\right\|_{L^{2}\left(\omega_{K}\right)^{3}} .
\end{aligned}
$$


From Lemma 1 we know that there exist some $z_{N+1} \in V_{0}^{p}\left(\left.\mathcal{K}_{N+1}\right|_{\omega_{K}}, \omega_{K}\right)$, $\zeta_{N+1} \in X_{0}^{p}\left(\left.\mathcal{K}_{N+1}\right|_{\omega_{K}}, \omega_{K}\right)^{3}$ and $p_{N+1} \in X_{0}^{p}\left(\left.\mathcal{K}_{N+1}\right|_{\omega_{K}}, \omega_{K}\right)$ such that

$$
\phi_{N+1}=z_{N+1}+\Pi_{N+1}^{\text {curl }} \zeta_{N+1}+\nabla p_{N+1}
$$

with $\Pi_{N+1}^{\text {curl }}: H_{0}(\operatorname{curl}, \Omega) \cap H^{r}(\Omega)^{3} \rightarrow V_{0}^{p}\left(\mathcal{K}_{N+1}, \Omega\right)$ as in Theorem 2. Choosing $\phi_{N}:=\Pi_{N}^{\text {curl }} \zeta_{N+1}$ with $\Pi_{N}^{\text {curl }}: H_{0}(\operatorname{curl}, \Omega) \cap H^{r}(\Omega)^{3} \rightarrow V_{0}^{p}\left(\mathcal{K}_{N}, \Omega\right)$ as in Theorem 2 implies

$$
\begin{aligned}
& \left\|\phi_{N+1}-\phi_{N}\right\|_{L^{2}\left(\omega_{K}\right)^{3}} \\
& \quad=\left\|z_{N+1}+\Pi_{N+1}^{\text {curl }} \zeta_{N+1}+\nabla p_{N+1}-\Pi_{N}^{\text {curl }} \zeta_{N+1}\right\|_{L^{2}\left(\omega_{K}\right)^{3}} \\
& \quad \leq\left\|z_{N+1}\right\|_{L^{2}\left(\omega_{K}\right)^{3}}+\left\|\nabla p_{N+1}\right\|_{L^{2}\left(\omega_{K}\right)^{3}}+\left\|\Pi_{N+1}^{\text {curl }} \zeta_{N+1}-\zeta_{N+1}\right\|_{L^{2}\left(\omega_{K}\right)^{3}} \\
& \quad \quad+\left\|\Pi_{N}^{\text {curl }} \zeta_{N+1}-\zeta_{N+1}\right\|_{L^{2}\left(\omega_{K}\right)^{3}}
\end{aligned}
$$

and by using Theorem 2 and regularity assumptions (4) and (11) it follows

$$
\begin{aligned}
\left\|\phi_{N+1}-\phi_{N}\right\|_{L^{2}\left(\omega_{K}\right)^{3}} \leq\left\|z_{N+1}\right\|_{L^{2}\left(\omega_{K}\right)^{3}}+\left\|\nabla p_{N+1}\right\|_{L^{2}\left(\omega_{K}\right)^{3}} \\
+2 C_{\text {curl }} \frac{h_{K}}{\left(p_{K}+1\right)^{1-\varepsilon}}\left\|\zeta_{N+1}\right\|_{H^{1}\left(\omega_{K, 1}\right)^{3} .}
\end{aligned}
$$

Then, Lemma 1 implies

$$
\left\|\phi_{N+1}-\phi_{N}\right\|_{L^{2}\left(\omega_{K}\right)^{3}} \leq C_{H} \frac{h_{K}}{\left(p_{K}+1\right)^{1-\varepsilon}}\left\|\nabla \times \phi_{N+1}\right\|_{L^{2}\left(\omega_{K, 1}\right)^{3}}
$$

for some constant $C_{H}>0$ independent of $h_{K}$ and $p_{K}$. Since $\operatorname{supp}\left(\phi_{N+1}\right) \subset$ $\omega_{K} \subset \omega_{K, 2}$, it follows

$$
\begin{aligned}
\left\|\phi_{N+1}-\phi_{N}\right\|_{L^{2}\left(\omega_{K}\right)^{3}} & \leq C_{H} \frac{h_{K}}{\left(p_{K}+1\right)^{1-\varepsilon}}\left\|\nabla \times \phi_{N+1}\right\|_{L^{2}\left(\omega_{K}\right)^{3}} \\
& \leq \frac{C_{H}}{\sqrt{C_{\text {ell }}}} \frac{h_{K}}{\left(p_{K}+1\right)^{1-\varepsilon}}\left\|\phi_{N+1}\right\|_{\omega_{K}}
\end{aligned}
$$

from (10). Inserting into (23) yields

$$
\begin{aligned}
& \left|\int_{\omega_{K}} \phi_{N+1}^{T} \operatorname{res}_{\omega_{K}}\right| \\
& \quad \leq\left(\left\|u_{N+1}-u_{N}\right\|_{\omega_{K}}+\frac{C_{H}}{\sqrt{C_{\text {ell }}}} \frac{h_{K}}{\left(p_{K}+1\right)^{1-\varepsilon}}\left\|f-f_{p_{\omega_{K}}+1}\right\|_{L^{2}\left(\omega_{K}\right)^{3}}\right)\left\|\phi_{N+1}\right\|_{\omega_{K}} .
\end{aligned}
$$

Then we divide by $\left\|\phi_{N+1}\right\|_{\omega_{K}}$ and take the supremum on both sides to obtain

$$
\begin{aligned}
& \sup _{\phi \in W_{0, K, j_{K}}^{p}}\left(\left.\mathcal{K}_{N}\right|_{\left.\omega_{K}, \omega_{K} ; \beta\right)}\left(\frac{\int_{\omega_{K}} \phi^{T} \operatorname{res}_{\omega_{K}}}{\|\phi\|_{\omega_{K}}}\right)\right. \\
& \leq \sup _{\substack{\phi_{N+1} \in W_{0}^{p}\left(\mathcal{K}_{N+1}, \Omega ; \beta\right) \\
\operatorname{supp}\left(\phi_{N+1}\right) \subset \omega_{K}}}\left(\frac{\int_{\omega_{K}} \phi_{N+1}^{T} \operatorname{res}_{\omega_{K}}}{\left\|\phi_{N+1}\right\|_{\omega_{K}}}\right) \\
& \leq\left\|u_{N+1}-u_{N}\right\|_{\omega_{K}}+\frac{C_{H}}{\sqrt{C_{e l l}}} \frac{h_{K}}{\left(p_{K}+1\right)^{1-\varepsilon}}\left\|f-f_{p_{\omega_{K}}+1}\right\|_{L^{2}\left(\omega_{K}\right)^{3}}
\end{aligned}
$$


and with (14) we have

$$
\varepsilon_{K, j_{K}} \eta_{K} \leq\left\|u_{N+1}-u_{N}\right\|_{\omega_{K}}+\frac{C_{H}}{\sqrt{C_{\text {ell }}}} \frac{h_{K}}{\left(p_{K}+1\right)^{1-\varepsilon}}\left\|f-f_{p_{\omega_{K}}+1}\right\|_{L^{2}\left(\omega_{K}\right)^{3}} .
$$

Squaring both sides and summing up over all $K \in \mathcal{A}_{N}$ yields

$$
\begin{aligned}
\sum_{K \in \mathcal{A}_{N}}\left(\varepsilon_{K, j_{K}} \eta_{K}\right)^{2} \leq 2( & \sum_{K \in \mathcal{K}_{N}}\left\|u_{N+1}-u_{N}\right\|_{\omega_{K}}^{2} \\
& \left.+\frac{C_{H}^{2}}{C_{\text {ell }}} \sum_{K \in \mathcal{K}_{N}} \frac{h_{K}^{2}}{\left(p_{K}+1\right)^{2(1-\varepsilon)}}\left\|f-f_{p_{\omega_{K}}+1}\right\|_{L^{2}\left(\omega_{K}\right)^{3}}^{2}\right),
\end{aligned}
$$

since $\mathcal{A}_{N} \subset \mathcal{K}_{N}$. With regularity assumptions (4) and (11) and the minimal property of $f_{p_{\omega_{K}}+1}$ in $L^{2}(K)^{3}$ for all $K \subset \omega_{K}$ this implies

$$
\begin{aligned}
\sum_{K \in \mathcal{A}_{N}}\left(\varepsilon_{K, j_{K}} \eta_{K}\right)^{2} \leq 2 C_{\text {cov }}( & \left\|u_{N+1}-u_{N}\right\|_{\Omega}^{2} \\
& \left.+\frac{C_{H}^{2}}{C_{e l l}} \sum_{K \in \mathcal{K}_{N}} \frac{h_{K}^{2}}{\left(p_{K}+1\right)^{2(1-\varepsilon)}}\left\|f-f_{p_{K}+1}\right\|_{L^{2}(K)^{3}}^{2}\right),
\end{aligned}
$$

where the covering constant $C_{c o v}>0$ is given by

$$
C_{\text {cov }}:=\max _{K \in \mathcal{K}_{N}}\left|\left\{L \in \mathcal{K}_{N}: L \subset \omega_{K}\right\}\right|
$$

with $|A|$ denoting the cardinality of set $A$. Finally by assumption (21) we get

$$
\sum_{K \in \mathcal{A}_{N}}\left(\varepsilon_{K, j_{K}} \eta_{K}\right)^{2} \leq 2 C_{c o v}\left(\left\|u_{N+1}-u_{N}\right\|_{\Omega}^{2}+\frac{C_{H}^{2}}{C_{e l l}} \mu^{2} \eta^{2}\right) .
$$

From Theorem 1 we know that there exists some constant $C_{1}>0$ independent of $h_{K}$ and $p_{K}$ such that

$$
\begin{aligned}
\left\|u-u_{N}\right\|_{\Omega}^{2} & \leq C_{1} \sum_{K \in \mathcal{K}_{N}}\left(p_{K}+1\right)^{2 \varepsilon}\left(\eta_{K}^{2}+\frac{h_{K}^{2}}{\left(p_{K}+1\right)^{2}}\left\|f-f_{p_{K}+1}\right\|_{L^{2}(K)^{3}}^{2}\right) \\
& \leq C_{1}\left(1+\mu^{2}\right) \max _{K \in \mathcal{K}_{N}}\left(p_{K}+1\right)^{2 \varepsilon} \eta^{2}
\end{aligned}
$$

by (21) and with the additional assumption $\mu \leq 1$ we obtain

$$
\theta^{2}\left\|u-u_{N}\right\|_{\Omega}^{2} \leq 2 C_{1} \max _{K \in \mathcal{K}_{N}}\left(p_{K}+1\right)^{2 \varepsilon} \theta^{2} \eta^{2}
$$

for some $\theta \in(0,1]$. With constraint (17) we have

$$
\theta^{2}\left\|u-u_{N}\right\|_{\Omega}^{2} \leq 2 C_{1} \sum_{K \in \mathcal{A}_{N}}\left(\varepsilon_{K, j_{K}} \eta_{K}\right)^{2}
$$


and we see

$$
\theta^{2}\left\|u-u_{N}\right\|_{\Omega}^{2} \leq 4 C_{1} C_{\text {cov }}\left(\left\|u_{N+1}-u_{N}\right\|_{\Omega}^{2}+\frac{C_{H}^{2}}{C_{\text {ell }}} \mu^{2} \eta^{2}\right)
$$

by inserting estimate (25). From Theorem 1 we also know

$$
\begin{aligned}
& \eta^{2} \leq C_{2}(\varepsilon) \sum_{K \in \mathcal{K}_{N}}\left(p_{K}+1\right)^{2(2+\varepsilon)}(\| u\left.-u_{N}\left\|_{\omega_{K}}^{2}+\frac{h_{K}^{2}}{\left(p_{K}+1\right)^{2+\varepsilon}}\right\| f-f_{p_{K}+1} \|_{L^{2}\left(\omega_{K}\right)^{3}}^{2}\right) \\
& \leq C_{2}(\varepsilon) C_{\operatorname{cov}} \max _{K \in \mathcal{K}_{N}}\left(p_{K}+1\right)^{2(2+\varepsilon)}\left(\left\|u-u_{N}\right\|_{\Omega}^{2}\right. \\
&\left.+\sum_{K \in \mathcal{K}_{N}} \frac{h_{K}^{2}}{\left(p_{K}+1\right)^{2(1-\varepsilon)}}\left\|f-f_{p_{K}+1}\right\|_{L^{2}(K)}^{2}\right)
\end{aligned}
$$

and with data regularity assumption (21) it follows

$$
\eta^{2} \leq C_{2}(\varepsilon) C_{\operatorname{cov}} \max _{K \in \mathcal{K}}\left(p_{K}+1\right)^{2(2+\varepsilon)}\left(\left\|u-u_{N}\right\|_{\Omega}^{2}+\mu^{2} \eta^{2}\right) .
$$

Hence for

$$
\mu<\frac{1}{\sqrt{C_{2}(\varepsilon) C_{\operatorname{cov}}} \max _{K \in \mathcal{K}_{N}}\left(p_{K}+1\right)^{2+\varepsilon}}
$$

we have

$$
\eta^{2}<\frac{C_{2}(\varepsilon) C_{\operatorname{cov}} \max _{K \in \mathcal{K}_{N}}\left(p_{K}+1\right)^{2(2+\varepsilon)}}{1-C_{2}(\varepsilon) C_{\operatorname{cov}} \max _{K \in \mathcal{K}_{N}}\left(p_{K}+1\right)^{2(2+\varepsilon)} \mu^{2}}\left\|u-u_{N}\right\|_{\Omega}^{2}
$$

and with the more restrictive assumption

$$
\mu \leq \frac{1}{\sqrt{2 C_{2}(\varepsilon) C_{\text {cov }}} \max _{K \in \mathcal{K}_{N}}\left(p_{K}+1\right)^{2+\varepsilon}}
$$

we get

$$
\eta^{2} \leq 2 C_{2}(\varepsilon) C_{\text {cov }} \max _{K \in \mathcal{K}_{N}}\left(p_{K}+1\right)^{2(2+\varepsilon)}\left\|u-u_{N}\right\|_{\Omega}^{2} .
$$

Inserting into (26) yields

$$
\begin{aligned}
& \theta^{2}\left\|u-u_{N}\right\|_{\Omega}^{2} \\
& \quad \leq 4 C_{1} C_{\text {cov }}\left(\left\|u_{N+1}-u_{N}\right\|_{\Omega}^{2}+\frac{2 C_{H}^{2} C_{2}(\varepsilon) C_{\text {cov }}}{C_{\text {ell }}} \max _{K \in \mathcal{K}_{N}}\left(p_{K}+1\right)^{2(2+\varepsilon)} \mu^{2}\left\|u-u_{N}\right\|_{\Omega}^{2}\right)
\end{aligned}
$$

and with the assumption

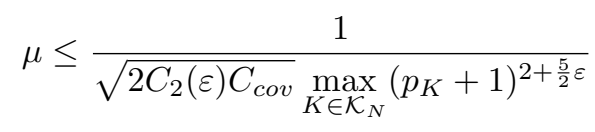


we obtain

$\theta^{2}\left\|u-u_{N}\right\|_{\Omega}^{2} \leq 4 C_{1} C_{\text {cov }}\left(\left\|u_{N+1}-u_{N}\right\|_{\Omega}^{2}+\frac{C_{H}^{2}}{C_{\text {ell }}} \max _{K \in \mathcal{K}_{N}}\left(p_{K}+1\right)^{-3 \varepsilon}\left\|u-u_{N}\right\|_{\Omega}^{2}\right)$.

Therefore

$$
\frac{1}{4 C_{1} C_{\text {cov }}}\left(\theta^{2}-\frac{4 C_{1} C_{\text {cov }} C_{H}^{2}}{C_{e l l} \max _{K \in \mathcal{K}_{N}}\left(p_{K}+1\right)^{3 \varepsilon}}\right)\left\|u-u_{N}\right\|_{\Omega}^{2} \leq\left\|u_{N+1}-u_{N}\right\|_{\Omega}^{2}
$$

and by assumption (19) we have

$$
\theta^{2}-\frac{4 C_{1} C_{c o v} C_{H}^{2}}{C_{e l l} \max _{K \in \mathcal{K}_{N}}\left(p_{K}+1\right)^{3 \varepsilon}} \geq \frac{\theta^{2}}{5}
$$

Since $C_{\text {cov }}>1$ and $\theta \leq \sqrt{20 C_{1}}$, inequality (22) holds with

$$
\kappa^{2}:=1-\frac{\theta^{2}}{20 C_{1} C_{c o v}}
$$

This completes the proof.

Remark 4. 1. For $\theta \in\left(0, \min \left\{1, \sqrt{20 C_{1}}\right\}\right]$ assumption (19) can always be satisfied by choosing $p_{K}$ large enough for some $K \in \mathcal{K}_{N}$.

2. Data regularity assumption (21) can only be satisfied, if the integrals on the left-hand side are computed with negligible error. To achieve this one can use high-order quadrature rules or perform local refinement according to the local interpolation error $\left\|f-f_{p_{K}+1}\right\|_{L^{2}(K)^{3}}$ until (21) is satisfied. Another option is to build some data error control into the whole algorithm as proposed in [18, 20].

Note, since estimates (12) and (13) are not uniform with respect to the polynomial degree $p_{K}$, data regularity assumption (21) becomes more and more restrictive for increasing $p_{K}$.

3. If the convergence indicators $\varepsilon_{K, j_{K}}$ are too small or $\varepsilon$ and $\theta$ are chosen too large, then constraint (17) cannot be satisfied and thus no solution of maximization problem (16), (17) exists. Especially this is the case, if

$$
\max _{K \in \mathcal{K}_{N}}\left(\varepsilon_{K, j_{K}}\right)<\max _{K \in \mathcal{K}_{N}}\left(p_{K}+1\right)^{\varepsilon} \theta
$$

Then our algorithm will continue with global h-refinement to enforce at least some convergence. If $\varepsilon_{K, j_{K}}$ is uniformly bounded from below, $\varepsilon$ and $\theta$ can be chosen such that uniform convergence is assured due to Theorem 3. This might be shown by following the ideas of Theorem 5 in [14]. A practical approach might be to monitor the computed values of $\varepsilon_{K, j_{K}}$ and thus check convergence in an a posteriori way. 
4. Our analysis does not guarantee optimality of the algorithm, but our convergence result might be a first step towards proving optimality [4, 24]. The difficulty lies in the maximization of (16) and its connection with approximation properties of solutions. However, we can observe that our refinement strategy performs well for the class of problems, which we consider here. Especially we did not observe any lock-up of the refinement algorithm due to a series of inefficient refinements as long as assumption (20) was satisfied.

\section{Numerical Examples}

In this section we apply our fully automatic $h p$-adaptive refinement strategy to some representative problems of the form (7). All computations are performed with the finite element library deal.II $[1,2]$.

\subsection{Example 1}

In our first example we consider a problem with a smooth solution. Let $\Omega:=(0,1)^{3}, \alpha(x):=\left(\sin \left(2 \pi x_{1}\right) \sin \left(2 \pi x_{2}\right) \sin \left(2 \pi x_{3}\right)+1.5\right) I$ and $\beta:=I$. The solution $u$ is given by

$$
u(x):=\left(\begin{array}{c}
0 \\
0 \\
\sin \left(\pi x_{1}\right)
\end{array}\right) .
$$

We start our algorithm with an initial triangulation $\mathcal{K}_{0}$ consisting of 8 cells and polynomial degree vector $p=0$. As refinement patterns we offer bisection in every coordinate-direction as $h$-refinement and increase of the polynomial degree by one as $p$-refinement. For later reference we denote this strategy by $\Theta_{1}$. Further we choose $\varepsilon=10^{-5}$ and $\theta=0.9$. In Figure 3 on the left-hand side we plot the number of degrees of freedom vs. the energy error. On the right-
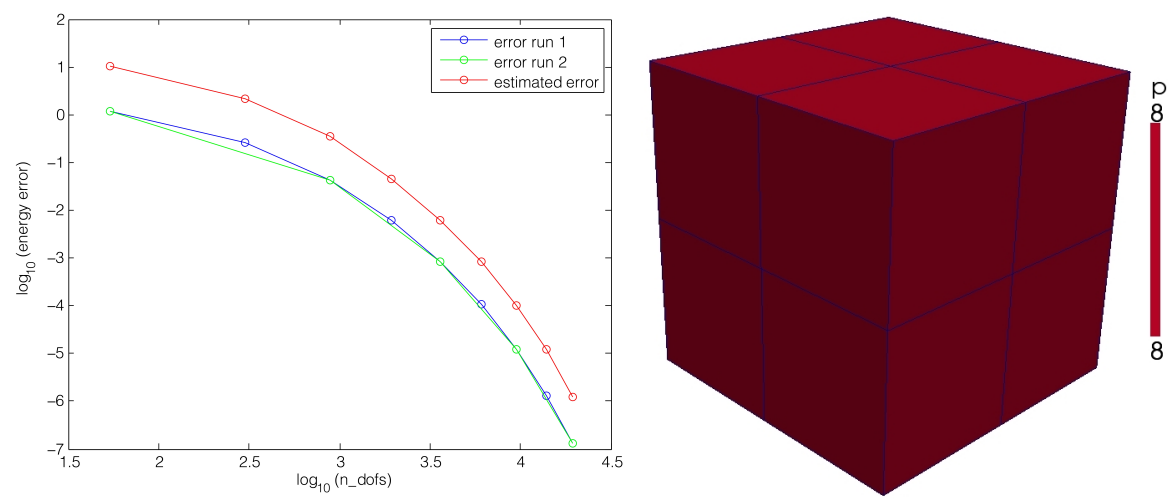

Figure 3: Example 1. Left: Number of degrees of freedom vs. energy error. Right: Final grid.

hand side the final grid after refinement step 7 is shown. The refinement history 


$\begin{array}{cccccccc}\text { Step } & \max (p) & \# h & \# p & & & & \\ 0 & 0 & 0 & 8 & & & & \\ 1 & 1 & 0 & 8 & \text { Step } & \max (p) & \# h & \# p \\ 2 & 2 & 0 & 8 & 0 & 0 & 0 & 8 \\ 3 & 3 & 0 & 8 & 1 & 2 & 0 & 8 \\ 4 & 4 & 0 & 8 & 2 & 4 & 0 & 8 \\ 5 & 5 & 0 & 8 & 3 & 6 & 0 & 8 \\ 6 & 6 & 0 & 8 & & & & \\ 7 & 7 & 0 & 8 & & & & \end{array}$

Table 1: Example 1: Refinement history. Left: Strategy $\Theta_{1}$. Right: Strategy $\Theta_{2}$.

can be seen in Table 1 on the left-hand side. Here \# $h$ denotes the number of $h$-refinements and $\# p$ the number of $p$-refinements, which were performed in the refinement step. Since in this example there are no local features to detect and the solution is smooth, the algorithm chooses global $p$-refinement in every refinement step.

In a second run we additionally provide the possibility to increase the polynomial degree by two. This strategy is denoted by $\Theta_{2}$. In Table 1 on the right-hand side the refinement history of this strategy can be seen. We observe that our algorithm takes profit from the additional possibility to increase the polynomial degree by two and, hence, reduces the number of refinement steps required to obtain the final grid. This also pays out in the total computation time. Whereas the first run took 11:12 minutes the second one took only 5:01 minutes on one node with 24 cores and 64 GB of total memory.

\subsection{Example 2}

In this example we choose again $\Omega:=(0,1)^{3}$, but $\alpha(x):=I$ for all $x \in \Omega$ and

$$
\beta(x):=\left\{\begin{array}{ll}
I \quad, \text { if } \max _{i \in\{1,2,3\}}\left|x_{i}-0.5\right| \leq 0.25 \\
0 \quad, \text { else }
\end{array} .\right.
$$

The solution $u$ is given by (27). This example already has the usual geometry of a realistic electromagnetic problem, namely a conducting region, where $\beta \neq 0$ holds, and a nonconducting region, where $\beta=0$ holds. Note that $\beta$ does not fit into our analytical setting, because it is not uniformly positive definite. Thus we have to replace $\beta$ by

$$
\chi_{\delta}(\beta):= \begin{cases}\delta I, & \text { if } \min _{\substack{u \in L^{2}\left(\omega_{K}\right)^{3} \\ u \neq 0}}\left(\frac{\int_{\omega_{K}}\left|u^{T} \beta u\right|}{\|u\|_{L^{2}\left(\omega_{K}\right)^{3}}}\right)<\delta \\ \beta, & \text { else }\end{cases}
$$

for some $\delta>0$ with $\delta \ll 1$. With this modification we are back in our analytical background, since $\chi_{\delta}(\beta)$ now satisfies assumption (6) and problem (15) is uniquely solvable on all patches $\omega_{K}$. We start our computation with a coarse 
grid $\mathcal{K}_{0}$ consisting of 64 equal-sized cells and polynomial degree vector $p=0$. As in Example 1 we try both strategies, $\Theta_{1}$ and $\Theta_{2}$, in two different runs. To be able to compare these two strategies fairly well we choose $\theta:=0.9$ quite big, $\varepsilon=10^{-5}$ and $\delta=10^{-10}$. In Figure 4 on the left-hand side we plot the number of degrees of freedom vs. the energy error. Also in this example we observe that
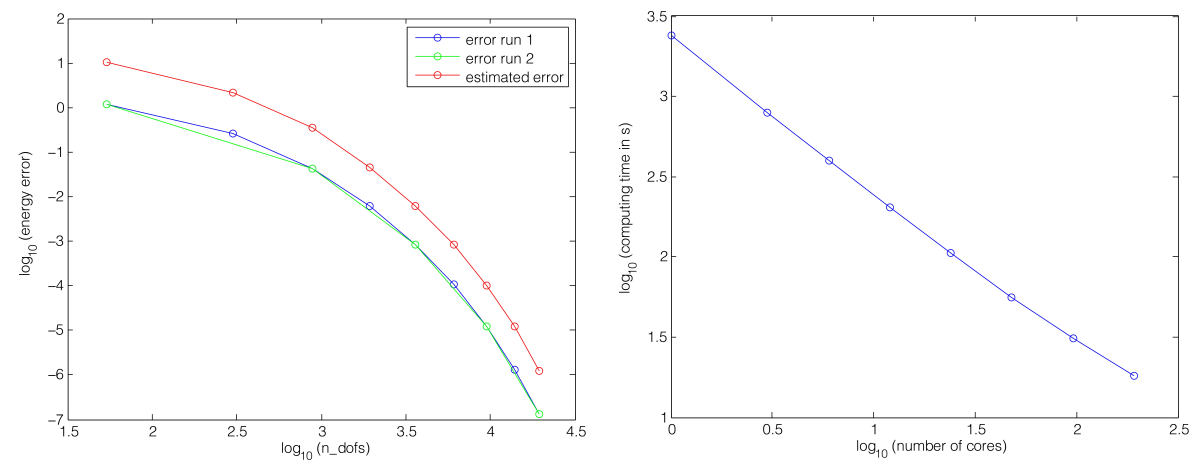

Figure 4: Example 2: Left: Number of degrees of freedom vs. energy error. Right: Scaling of computing time for refinement patterns.

the fully automatic $h p$-adaptive refinement algorithm recognizes the smoothness of the solution and performs $p$-refinement only. On the right-hand side of Figure 4 we have plotted the average computational time, which is required to compute the convergence indicators $\varepsilon_{j, K}$ from Section 3.3, in strategy $\Theta_{2}$ for a varying number of CPU cores. We observe that the computation time decreases with growing number of cores.

\subsection{Example 3}

In this example we choose almost the same setting as in Example 2. Let $\Omega, \alpha, \beta$ and $\chi_{\delta}(\beta)$ be as above. Further let $f=1$ and $g=0$. By this choice the solution $u$ becomes singular and we finally have a more realistic example. The analytic solution $u$ of this problem is unknown. We start with the same grid and polynomial degree vector as in Example 2. Further we choose $\varepsilon=$ $10^{-5}, \delta=10^{-10}$ and $\theta=0.275$. In Figure 5 we plot the number of degrees of freedom vs. the estimated error. Also in this example the algorithm captures the edge singualrities quite well and performs $h$-refinement around the edges of the nonconducting regions (cf. Figure 5). The refinement history can be seen in Table 2 .

\subsection{Example 4}

In the last example we solve a classical academic problem. Let $\Omega:=(-1,1)^{3} \backslash$ $([0,1) \times(-1,0] \times(-1,1))$ and $\alpha:=\beta:=I$. The analytic solution of this problem 

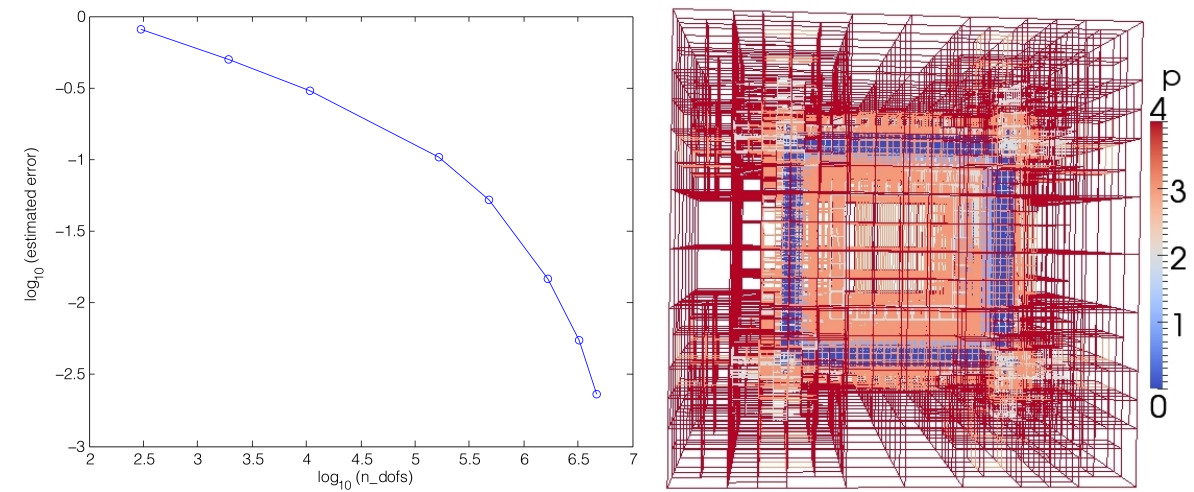

Figure 5: Example 3: Left: Number of degrees of freedom vs. estimated error. Right: Final grid.

$\begin{array}{cccc}\text { Step } & \max (p) & \# h & \# p \\ 0 & 0 & 64 & 0 \\ 1 & 0 & 256 & 64 \\ 2 & 1 & 988 & 156 \\ 3 & 2 & 36 & 296 \\ 4 & 2 & 4056 & 32 \\ 5 & 3 & 15128 & 725 \\ 6 & 3 & 57112 & 3703\end{array}$

Table 2: Example 3: Refinement history.

is given by

$$
u\left(r, \phi, x_{3}\right):=\frac{2}{3} r^{-\frac{1}{3}}\left(\begin{array}{c}
-\sin \left(\frac{\phi}{3}\right) \\
\cos \left(\frac{\phi}{3}\right) \\
0
\end{array}\right),
$$

where $r \in \mathbb{R}_{+}$and $\phi \in[0,2 \pi)$ denote the polar coordinates. We start the algorithm with an initial grid $\mathcal{K}_{0}$ consisting of 48 cells and polynomial degree vector $p=0$. For the parameters $\varepsilon>0$ and $\theta \in(0,1]$ we choose $\varepsilon=10^{-5}$ and $\theta=0.2$. We plot the number of degrees of freedom vs. the energy error in Figure 6 on the left-hand side. On the right-hand side the $\left(x_{1}, x_{2}\right)$-cut of the final grid is shown. Also in this example the algorithm recognizes the edge singularity along the corner edge $\left(0,0, x_{3}\right)$ quite well.

\section{Conclusion}

We have shown a fully automatic $h p$-adaptive refinement strategy for Maxwell's equations in the electric field formulation. Further we have proven its convergence and gave some numerical examples to see how the refinement strategy performs under different circumstances. 

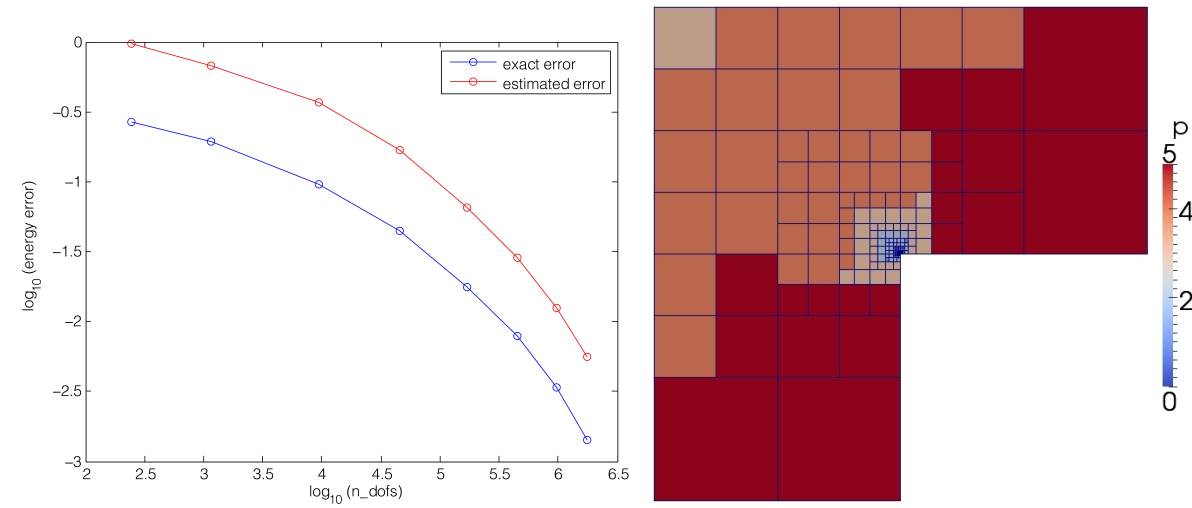

Figure 6: Example 4: Left: Number of degrees of freedom vs. energy error. Right: Final grid.

\section{Acknowledgements}

The author wants to thank Willy Dörfler for many valueable comments and fruitful discussions about this work.

\section{References}

[1] W. Bangerth, R. Hartmann, and G. Kanschat. deal.II - a general purpose object oriented finite element library. ACM Trans. Math. Softw., $33(4): 24 / 1-24 / 27,2007$.

[2] W. Bangerth and G. Kanschat. deal.II Differential Equations Analysis Library, Technical Reference. http://www.dealii.org.

[3] R. Beck, R. Hiptmair, R.H.W. Hoppe, and B. Wohlmuth. Residual based a posteriori error estimators for eddy current computation. M2AN, 34(1):159-182, 2000.

[4] P. Binev, W. Dahmen, and R. DeVore. Adaptive finite element methods with convergence rates. Numer. Math., 97:219-268, 2004.

[5] M. Bürg. An hp-Efficient Residual-Based A Posteriori Error Estimator for Maxwell's Equations. Preprint 11/05, IWRMM, Karlsruhe Institute of Technology, 2011.

[6] M. Bürg and W. Dörfler. Convergence of an adaptive $h p$ finite element strategy in higher space-dimensions. Appl. Numer. Math., 61:1132-1146, 2011.

[7] J. Chen, Y. Xu, and J. Zou. Convergence analysis of an adaptive edge element method for Maxwell's equations. Appl. Numer. Math., 59:29502969, 2009. 
[8] Th.H. Cormen, C.E. Leiserson, R. Rivest, and C. Stein. Introduction to Algorithms. The MIT Press, Cambridge, MA, 2001.

[9] M. Costabel, M. Dauge, and S. Nicaise. Singularities of Maxwell interface problems. M2AN, 33(3):627-649, 1999.

[10] L. Demkowicz. Fully automatic $h p$-adaptivity for Maxwell's equations. Comput. Meth. Appl. Mech. Engrg., 194:605-624, 2005.

[11] L. Demkowicz and A. Buffa. $H^{1}, H$ (curl) and $H$ (div)-conforming projection-based interpolation in three dimensions: Quasi-optimal $p$ interpolation estimates. Comput. Meth. Appl. Mech. Engrg., 194:267-296, 2005 .

[12] L. Demkowicz, J. Kurtz, D. Pardo, M. Paszyński, W. Rachowicz, and A. Zdunek. Computing with hp-Adaptive Finite Elements, volume 2. Chapman\&Hall/CRC, Boca Raton, FL, 2008.

[13] W. Dörfler. A Convergent Adaptive Algorithm for Poisson's Equation. SIAM J. Numer. Anal., 33(3):1106-1124, 1996.

[14] W. Dörfler and V. Heuveline. Convergence of an adaptive $h p$ finite element strategy in one space dimension. Appl. Numer. Math., 57:1108-1124, 2007.

[15] W. Dörfler, A. Lechleiter, M. Plum, G. Schneider, and C. Wieners. Photonic Crystals: Mathematical Analysis and Numerical Approximation. Springer, Basel, 2011.

[16] D. Harutyunyan, F. Izsák, J.J.W. van der Vegt, and M.A. Botchev. Adaptive finite element techniques for the Maxwell equations using implicit a posteriori error estimates. Comput. Meth. Appl. Mech. Engrg., 197:1620$1638,2008$.

[17] R. Hiptmair and J. Xu. Nodal auxiliary space preconditioning in $H$ (curl) and $H($ div) spaces. SIAM J. Numer. Anal., 45:2483-2509, 2007.

[18] K. Mekchay and R.H. Nochetto. Convergence of adaptive finite element methods for general second order elliptic PDEs. SIAM J. Numer. Anal., 43(5):1803-1827, 2005.

[19] P. Monk. Finite Element Methods for Maxwell's Equations. Clarendon Press, Oxford, 2003.

[20] P. Morin, R.H. Nochetto, and K.G. Siebert. Data oscillation and convergence of adaptive FEM. SIAM J. Numer. Anal., 38(2):466-488, 2000.

[21] D. Pardo. Integration of hp-adaptivity with a two grid solver: applications to electromagnetics. PhD thesis, The University of Texas at Austin, April 2004 . 
[22] D. Pardo, L. Demkowicz, and J. Gopalakrishnan. Integration of $h p$ adaptivity and a two grid solver for electromagnetic problems. Comput. Meth. Appl. Mech. Engrg., 195:2533-2573, 2005.

[23] Ch. Schwab. p- and hp-Finite Element Methods. Clarendon Press, Oxford, 1998.

[24] R. Stevenson. Optimality of a standard adaptive finite element method. Found. Comput. Math., 7(2):245-269, 2007.

[25] B. Szabó and I. Babuška. Finite Element Analysis. Wiley, New York, NY, 1991. 


\section{IWRMM-Preprints seit 2009}

Nr. 09/01 Armin Lechleiter, Andreas Rieder: Towards A General Convergence Theory For Inexact Newton Regularizations

Nr. 09/02 Christian Wieners: A geometric data structure for parallel finite elements and the application to multigrid methods with block smoothing

Nr. 09/03 Arne Schneck: Constrained Hardy Space Approximation

Nr. 09/04 Arne Schneck: Constrained Hardy Space Approximation II: Numerics

Nr. 10/01 Ulrich Kulisch, Van Snyder : The Exact Dot Product As Basic Tool For Long Interval Arithmetic

Nr. 10/02 Tobias Jahnke : An Adaptive Wavelet Method for The Chemical Master Equation

Nr. 10/03 Christof Schütte, Tobias Jahnke : Towards Effective Dynamics in Complex Systems by Markov Kernel Approximation

Nr. 10/04 Tobias Jahnke, Tudor Udrescu : Solving chemical master equations by adaptive wavelet compression

Nr. 10/05 Christian Wieners, Barbara Wohlmuth : A Primal-Dual Finite Element Approximation For A Nonlocal Model in Plasticity

Nr. 10/06 Markus Bürg, Willy Dörfler: Convergence of an adaptive hp finite element strategy in higher space-dimensions

Nr. 10/07 Eric Todd Quinto, Andreas Rieder, Thomas Schuster: Local Inversion of the Sonar Transform Regularized by the Approximate Inverse

Nr. 10/08 Marlis Hochbruck, Alexander Ostermann: Exponential integrators

Nr. 11/01 Tobias Jahnke, Derya Altintan : Efficient simulation of discret stochastic reaction systems with a splitting method

Nr. 11/02 Tobias Jahnke : On Reduced Models for the Chemical Master Equation

Nr. 11/03 Martin Sauter, Christian Wieners : On the superconvergence in computational elastoplasticity

Nr. 11/04 B.D. Reddy, Christian Wieners, Barbara Wohlmuth : Finite Element Analysis and Algorithms for Single-Crystal Strain-Gradient Plasticity

Nr. 11/05 Markus Bürg: An hp-Efficient Residual-Based A Posteriori Error Estimator for Maxwell's Equations

Nr. 12/01 Branimir Anic, Christopher A. Beattie, Serkan Gugercin, Athanasios C. Antoulas: Interpolatory Weighted-H2 Model Reduction

Nr. 12/02 Christian Wieners, Jiping Xin: Boundary Element Approximation for Maxwell's Eigenvalue Problem

Nr. 12/03 Thomas Schuster, Andreas Rieder, Frank Schöpfer: The Approximate Inverse in Action IV: Semi-Discrete Equations in a Banach Space Setting

Nr. 12/04 Markus Bürg: Convergence of an hp-Adaptive Finite Element Strategy for Maxwell's Equations

Eine aktuelle Liste aller IWRMM-Preprints finden Sie auf: 


\section{Kontakt}

Karlsruher Institut für Technologie (KIT) Institut für Wissenschaftliches Rechnen und Mathematische Modellbildung

Prof. Dr. Christian Wieners Geschäftsführender Direktor

Campus Süd

Engesserstr. 6

76131 Karlsruhe

E-Mail:Bettina.Haindl@kit.edu

www. math. kit.edu/iwrmm/

\section{Herausgeber}

Karlsruher Institut für Technologie (KIT) Kaiserstraße 12 | 76131 Karlsruhe

März 2012 